\title{
Exact solution of a nonlinear model of four-wave mixing and phase conjugation
}

\author{
Mark Cronin-Golomb, Jeffrey O. White, Baruch Fischer, and Amnon Yariv \\ California Institute of Technology, Pasadena, California 91125
}

Received March 29, 1982

\begin{abstract}
An exact solution of a nonlinear model of holographic four-wave mixing is derived. An expression for the reflectivity of a phase-conjugate mirror with depleted pumps is presented. We find that such a phase-conjugate mirror may exhibit bistability.
\end{abstract}

Theories of the interactions of light in media possessing optical nonlinearities are themselves strongly nonlinear; most theoretical approaches have depended on such linearizing assumptions as the undepleted-pumps approximation. ${ }^{1,2}$ Between the early 1960's, when second- and third-harmonic generation ${ }^{3}$ and Raman pump conversion to first Stokes line ${ }^{4}$ were solved, and the present, little work was done that analytically retained the nonlinear nature of the problem. In 1979, a model of degenerate four-wave mixing in isotropic media was solved for the case in which all four beams are collinear. ${ }^{5}$ As a result of recent progress in the search for nonlinear media that are efficient in coupling light at low power levels, four-wave mixing and phase-conjugation experiments in which the pumping beams cannot be considered undepleted are becoming more numerous. ${ }^{6-8}$ There is thus a more urgent need for solutions of theories of four-wave mixing that do not rely on the constancy of the pumps.

We present here such a solution, which is applicable in the case of degenerate four-wave mixing by real-time holography. ${ }^{9}$ This solution does not suffer from the collinearity restriction and is applicable for any phase shift between the refractive-index grating and the light-interference pattern. The basic interaction geometry is illustrated in Fig. 1. Four waves of equal frequency $\omega$, and of the same polarization, are propagating through the nonlinear medium. Let the electric-field amplitude associated with the $j$ th beam be

$$
E_{j}=A_{j}(\mathbf{r}) \exp \left[i\left(\mathbf{k}_{j} \cdot \mathbf{r}-\tilde{\omega} t\right)\right]+\text { c.c. }
$$

We solve the problem in the steady state, so that $A_{j}$ may be taken to be time independent. The propagation directions come in two oppositely directed pairs, $\mathbf{k}_{1}=$ $-\mathbf{k}_{2}$ and $\mathbf{k}_{3}=-\mathbf{k}_{4}$, and the angles between $\mathbf{k}_{1}$ and the crystal normal and $\mathbf{k}_{2}$ and the crystal normal are equal in magnitude but opposite in sign. A set of four nonlinear coupled-wave equations can be derived by using the standard slowly varying field approximation. ${ }^{10}$ We simplify the problem by considering a holographic system whose response is such that, of all the gratings present in the system, only one grating, in our case the one created by the interference of beam 1 with beam 4 and beam 2 with 3 , gives rise to strong beam coupling. This predominance of one grating is common in many laboratory situations and is due to the directions, polarizations, and coherence relationships of the four beams relative to the nonlinear medium and to the application, in some cases, of an electric field that enhances certain gratings. We do not use the undepleted-pumps approximation. That is, the variation of all four beams is retained, and the solution is valid even when all four beams are comparable in amplitude. The applicable coupled-wave equations are ${ }^{10}$

$$
\begin{aligned}
\frac{\mathrm{d} A_{1}}{\mathrm{~d} z} & =\frac{-\gamma}{I_{0}}\left(A_{1} A_{4} *+A_{2} * A_{3}\right) A_{4}, \\
\frac{\mathrm{d} A_{2}{ }^{*}}{\mathrm{~d} z} & =\frac{-\gamma}{I_{0}}\left(A_{1} A_{4} *+A_{2} * A_{3}\right) A_{3} *, \\
\frac{\mathrm{d} A_{3}}{\mathrm{~d} z} & =\frac{\gamma}{I_{0}}\left(A_{1} A_{4} *+A_{2} * A_{3}\right) A_{2}, \\
\frac{\mathrm{d} A_{4}^{*}}{\mathrm{~d} z} & =\frac{\gamma}{I_{0}}\left(A_{1} A_{4} *+A_{2} * A_{3}\right) A_{1} *
\end{aligned}
$$

where $\gamma$ is a complex coupling constant, which is a material parameter of the nonlinear medium. ${ }^{11}$ When $\gamma$ is real, the phase shift between grating and fringes is $\pi / 2$. When it is purely imaginary, the grating is in phase with the fringes. $I_{0}$ is the total average light intensity.

$$
I_{0}=I_{1}+I_{2}+I_{3}+I_{4},
$$

where $I_{j}=\left|A_{j}\right|^{2}$.

We observe the following conservation laws:

$$
\begin{aligned}
A_{1} A_{2}+A_{3} A_{4} & =c, \\
I_{1}+I_{4} & =d_{1}, \\
I_{2}+I_{3} & =d_{2},
\end{aligned}
$$

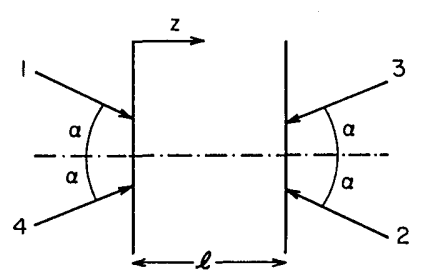

Fig. 1. Scheme of the four beams involved in nonlinear interaction. 
where $c, d_{1}$, and $d_{2}$ are constants of integration.

With the help of these conservation laws, Eqs. (2) and

(3) can be decoupled from Eqs. (4) and (5):

$$
\begin{aligned}
\frac{\mathrm{d} A_{1}}{\mathrm{~d} z} & =\frac{-\gamma}{I_{0}}\left[A_{1} d_{1}-A_{1}\left(I_{1}+I_{2}\right)+A_{2} * c\right], \\
\frac{\mathrm{d} A_{2}^{*}}{\mathrm{~d} z} & =\frac{-\gamma}{I_{0}}\left[A_{1} c^{*}-A_{2} *\left(I_{1}+I_{2}\right)+A_{2} * d_{2}\right], \\
\frac{\mathrm{d} A_{3}}{\mathrm{~d} z} & =\frac{\gamma}{I_{0}}\left[A_{3} d_{2}-A_{3}\left(I_{3}+I_{4}\right)+A_{4} * c\right], \\
\frac{\mathrm{d} A_{4}^{*}}{\mathrm{~d} z} & =\frac{\gamma}{I_{0}}\left[A_{3} c^{*}-A_{4} *\left(I_{3}+I_{4}\right)+A_{4} * d_{1}\right] .
\end{aligned}
$$

By eliminating the term in $I_{1}+I_{2}$ between Eqs. (10) and (11), and the term in $I_{3}+I_{4}$ between Eqs. (12) and (13), we find the following expressions for $A_{3} / A_{4} *$ and $A_{1} / A_{2}{ }^{*}$ :

$$
\begin{aligned}
& \frac{\mathrm{d}}{\mathrm{d} z}\left(\frac{A_{1}}{A_{2} *}\right)=\frac{-\gamma}{I_{0}}\left[c+\left(d_{1}-d_{2}\right)\left(\frac{A_{1}}{A_{2} *}\right)-c^{*}\left(\frac{A_{1}}{A_{2} *}\right)^{2}\right], \\
& \frac{\mathrm{d}}{\mathrm{d} z}\left(\frac{A_{3}}{A_{4} *}\right)=\frac{\gamma}{I_{0}}\left[c+\left(d_{2}-d_{1}\right)\left(\frac{A_{3}}{A_{4} *}\right)-c^{*}\left(\frac{A_{3}}{A_{4} *}\right)^{2}\right] .
\end{aligned}
$$

Noting that $I_{0}$ is constant because of the conservation laws, we see that these equations are immediately integrable:

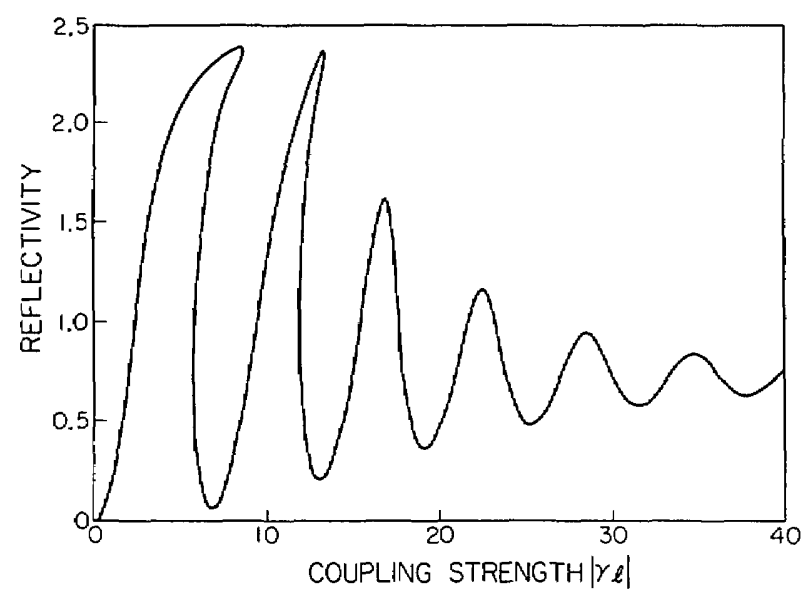

Fig. 2. Reflectivity of a phase-conjugate mirror versus coupling strength $|\gamma l|$. The incident pump beams are of equal intensity, the intensity of the incident probe beam is $20 \%$ of the total incident pumping intensity, and the phase shift between the index grating and interference fringes is $5^{\circ}$.

This solution, of course, reduces to the known undepleted-pumps approximation theory, ${ }^{10}$ in the limit of small $I_{4}(0)$. In Fig. 2 we plot the reflectivity of a phase-conjugate mirror as a function of the coupling strength $|\gamma l|$ for the case in which the phase shift be-

$$
\begin{aligned}
& \frac{A_{1}}{A_{2}{ }^{*}}=-\left\{\left[\frac{\left[\Delta-\left(\Delta^{2}+4|c|^{2}\right)^{1 / 2}\right] D e^{-\mu z}-\left[\Delta+\left(\Delta^{2}+4|c|^{2}\right)^{1 / 2}\right] D^{-1} e^{\mu z}}{2 c^{*}\left(D e^{-\mu z}-D^{-1} e^{\mu z}\right)}\right\},\right. \\
& \frac{A_{3}}{A_{4}{ }^{*}}=\left\{\left[\frac{\left[\Delta-\left(\Delta^{2}+4|c|^{2}\right)^{1 / 2}\right] E e^{-\mu z}-\left[\Delta+\left(\Delta^{2}+4|c|^{2}\right)^{1 / 2}\right] E^{-1} e^{\mu z}}{2 c^{*}\left(E e^{-\mu z}-E^{-1} e^{\mu z}\right)}\right\},\right.
\end{aligned}
$$

where $\Delta$ is the power flux $d_{2}-d_{1}$,

$$
\mu=\frac{\gamma\left(\Delta^{2}+4|c|^{2}\right)^{1 / 2}}{2 I_{0}},
$$

and $D$ and $E$ are constants of integration.

At this point, the problem has been transformed from the set of nonlinear differential equations [Eqs. (2)-(5)] to another set of equations [Eqs. (7)-(9), (16), and (17)], which may be solved by fitting boundary conditions.

To illustrate this solution in a particular set of boundary conditions, we consider the reflectivity of a phase-conjugate mirror. Let beams 1 and 2 be the pumping beams, beam 4 be the probe beam, and beam 3 be the phase-conjugate beam. $I_{1}(0), I_{4}(0)$, and $I_{2}(l)$ are given as boundary conditions, together with the phase-conjugation condition $I_{3}(l)=0$. After elementary manipulations of the solution equations and the boundary conditions, we find that the intensity reflectivity $R$ is given by

$$
R=\frac{4|c| 2|T|^{2}}{\left|\Delta T+\left(\Delta^{2}+4|c|^{2}\right)^{1 / 2}\right|^{2}}
$$

where $T=\tanh (\mu l)$ and $|c|^{2}$ is given by the equation

$$
\begin{gathered}
{\left[|c|^{2}-I_{1}(0) I_{2}(l)\right]\left|\Delta T+\left(\Delta^{2}+4|c|^{2}\right)^{1 / 2}\right|^{2}} \\
+4|c|^{2}|T|^{2} I_{4}(0) I_{2}(l) \\
\quad+2|c|^{2} I_{4}(0)\left(\Delta^{2}+4|c|^{2}\right)^{1 / 2}\left(T+T^{*}\right)=0 .
\end{gathered}
$$

tween the grating and the interference fringes is $5^{\circ}$. The intensities of the two pumping beams $I_{1}(0)$ and $I_{2}(l)$ have been taken to be equal, and the probe ratio $q$, defined as

$$
q=\frac{I_{4}(0)}{I_{1}(0)+I_{2}(l)}
$$

has been taken to be 0.2. The top of the graph $(R=2.5)$ corresponds to the reflectivity that would result if all the power of beam 2 were transferred to beam 3 . This is the maximum reflectivity consistent with the conservation laws [Eqs. (7)-(9)]. The peaks in the curve correspond to the poles in the reflectivity of a phase-conjugate mirror with no pump depletion $\left[R=|\tanh (\gamma l / 2)|^{2}\right.$ for no phase shift between grating and interference fringes]. In the graph, we have set the phase shift slightly nonzero to demonstrate the resultant damping of the oscillatory behavior. The peaks bend toward the right, probably because depletion causes high reflectivities to demand higher coupling strengths than are required for the same behavior in the undepleted phase-conjugate mirror. The bending of the peaks can even be sufficient for bistability, as can be seen in the first two peaks of Fig. 2.

In conclusion, then, we have solved a model of holographic four-wave mixing without using the unde- 
pleted-pumps approximation; the quantitative theoretical study of experiments involving pump depletion has thus been made possible without using numerical solutions of the coupled-wave differential equations.

The authors are grateful for valuable discussions with F. Vachss. M. Cronin-Golomb would like to acknowledge the support of the University of Sydney and B. Fischer the support of the Weizmann postdoctoral fellowship. This work was supported by the U.S. Air Force Office of Scientific Research and the U.S. Army Research Office, Durham, North Carolina.

\section{References}

1. A. Yariv and D. M. Pepper, Opt. Lett. 1, 16 (1977).

2. R. W. Hellwarth, J. Opt. Soc. Am. 67, 1 (1977).
3. J. A. Armstrong, N. Bloembergen, J. Ducuing, and P. S. Pershan, Phys. Rev. 127, 1918 (1962).

4. V. T. Platonenko and R. V. Khoklov, Zh. Eksp. Teor. Fiz. 46, 555 (1964) [Sov. Phys. JETP 19, 378 (1964)]:

5. J. H. Marburger and J. F. Lam, Appl. Phys. Lett. 34, 389 (1979); 35, 249 (1979).

6. J. Feinberg and R. W. Hellwarth, Opt. Lett. 5, 519 (1980).

7. R. C. Lind and D. G. Steel, Opt. Lett. 6, 554 (1981).

8. J. O. White, M. Cronin-Golomb, B. Fischer, and A. Yariv, Appl. Phys. Lett. 40, 450 (1982).

9. A. Yariv, Opt. Commun. 25, 23 (1978).

10. B. Fischer, M. Cronin-Golomb, J. O. White, and A. Yariv, Opt. Lett. 6, 519 (1981).

11. In photorefractive media, for example, $\gamma$ depends on parameters such as crystal orientation, grating period, charge-carrier concentration, and the angle of incidence $\alpha$. 